\title{
MOST PROBABLE PHASE DISTRIBUTION IN SPHERICAL STAR SYSTEMS AND CONDITIONS FOR ITS EXISTENCE
}

(orig.: Vest. Leningrad Univ. 7,135 ; 1962)

V. A. Antonov

The initial stages of the evolution of a spherical star system can be pictured in the following way. The stars have their beginning in a comparatively small region; the mechanism of the origin of initial velocities is not known exactly. From the point of view of the contemporary state of stellar astronomy, it is an acceptable view that the initial velocities have their origin in the attraction of each individual star towards the center of gravity of the system. The second possible assumption is to attribute the origin of initial velocities to forces of an explosive character. But in either case, initially, all orbits have to be almost exactly radial.

However, immediately after the formation of a spherical star system, irregular forces enter the picture. Such a process is investigated in the paper by T. A. Agekianl, where it is shown that in the initial phase many stars will escape from the central region. We shall consider the next phase, i.e. when in the central part a Maxwellian distribution of velocities has already been established. This distribution gradually propagates towards the periphery. In reality, the transition from the center to the periphery has to be continuous, but in order to simplify the computations, we shall suppose that when the evolution stops (more precisely, slows down strongly) it is possible to distinguish the main body of the star system from the corona." In the main body a Maxwellian distribution is established (we neglect the truncation of this distribution). In the corona, the original distribution of velocities is preserved. This means that there are no orbits which entirely avoid the main body.

Boltzmann's well-known theorem ${ }^{2}$ asserts that irregular forces can only increase the probability of the phase distribution and, consequent$1 y$, its logarithm (entropy). Boltzmann's theorem is proven under the assumptions that only binary collisions of an elastic character take place and that the interactions exhibited are central forces. However, this circumstance is not that essential. The reason is ${ }^{3}$ that close multiple encounters of stars are extremely rare phenomena, and multiple encounters at large distances are subject to the law of superposition, i.e. are equivalent to several consecutive binary passages. On the other hand, regular forces exhibit no influence on the 
entropy. From Boltzmann's theorem it immediately follows that the achievement of the maximum possible entropy is characteristic of the state of statistical equilibrium.

It is known from statistical mechanics that the state of statistical equilibrium (in the absence of rotation) has a Maxwelliam distribution as a necessary property at every point in space.

The aim of the present article is to show that this, generally speaking, is not sufficient, i.e. that a stationary state with a Maxwellian distribution of velocities is a state of statistical equilibrium only under certain conditions.

It has been remarked before that the region with a Maxwellian distribution in a real system is always bounded. Therefore, a stationary spherical star system with a Maxwellian distribution can be determined by three parameters: the radius, $\mathrm{R}$, of the Maxwellian region, the stellar number density at the center, $\nu_{C}$, and the root-mean-square velocity, $\sigma$. We are not, naturally, concerned with characteristics of individual stars. Escape of stars we neglect, proceeding as though the sphere at radius $R$ were a reflecting boundary.

Thus, we have to establish under what conditions the above-described phase distribution maximizes the entropy

$$
\mathrm{L}=-\iint \Psi \ln \Psi \mathrm{dr} \mathrm{dv}
$$

when the total mass, $M$, and total energy, $H$, are given. Strickly speaking, the total mass varies with time due to the fact that the system is not isolated from the surrounding world and may lose stars which reach a velocity greater than the escape velocity. But this process is comparatively slow and therefore is only of secondary importance for the configuration of a star system at any particular moment of time. ${ }^{4}$

Expressions for total mass and total energy

$$
\begin{gathered}
M=m \iint \Psi \mathrm{dr} d v \\
\mathrm{H}=\mathrm{M} \iint \Psi\left(\frac{\mathrm{v}^{2}}{2}+\frac{\Phi}{2}\right) \mathrm{dr} \mathrm{dv}=\mathrm{K}+\mathrm{W} .
\end{gathered}
$$

In formulas (1), (2) and (3) we use the following notations:

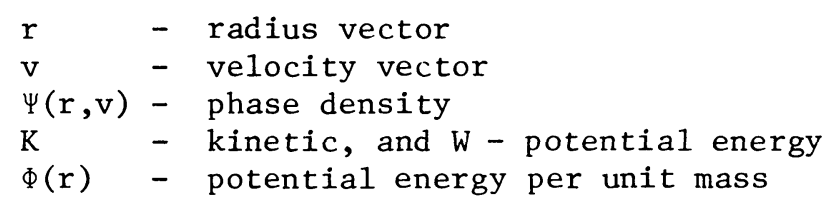

In (3) it is necessary to divide $\Phi$ by two in view of non-additivity of potential energy. If we took not one half, but the full potential, then in the integration over all stars, each pair of gravitational interactions would be counted twice, i.e. one would get $\mathrm{K}+2 \mathrm{~W}$.

The phase density of a stationary system will be designated by $\Psi_{0}$. 
The condition that $\mathrm{L}$ be maximized by $\Psi_{0}$ is equivalent to validity of the inequality

$$
\delta \mathrm{L}=\iint\left(\Psi_{0} \ln \Psi_{0}-\Psi \ln \Psi\right) \mathrm{dr} \mathrm{dv}<0
$$

for all permissible $\psi$ (absolute maximum) or only those close to $\Psi_{0}$ (relative maximum). We notice that the distinction between the relative and the absolute maximum corresponds in molecular physics to the distinction between metastable and fully-stable states.

In the beginning, we shall consider only the relative maximum. We shall introduce a certain classification of functions $\psi$. Namely, we shall consider $\Psi_{1}$ and $\Psi_{2}$ as belonging to one class if they have the same 1) star density $\nu(r)=\int \Psi \mathrm{dv}$, and hence also potential uniquely determined by the density;

2) kinetic energy $K$.

It is easy to see that all functions of one class are equally permissible or not permissible for a given $M$ and $H$. But within each class considerable freedom is left in the choice of $\Psi$ (e.g. different variations of velocity dispersion from point to point are possible). In each class, we choose a function $\psi$ to which corresponds the largest L. When such a choice is made, then validity of inequality (4) for the "worst case", $\Psi$, insures the validity for all the rest of the functions in the same class.

The selection process just described represents essentially an auxiliary constraint on the maximum, which we solve by Lagrange multipliers ${ }^{5}$

$$
\mathrm{L}+\int \lambda(\mathrm{r}) \nu(\mathrm{r}) \mathrm{dr}+\mu \mathrm{K}=\iint\left(-\Psi \ln \psi+\lambda(\mathrm{r}) \Psi+\frac{\mu \mathrm{v}^{2}}{2} \psi\right) \mathrm{dr} \mathrm{dv} .
$$

We find the variation of this expression with accuracy up to terms of second order

$$
\iint\left[-(\ln \Psi+1) \partial \Psi-\frac{(\partial \Psi)^{2}}{2 \Psi}+\lambda(r) \delta \Psi+\frac{\mu}{2} v^{2} \delta \Psi\right] d r d v .
$$

Equating to zero terms of first order, we obtain the solution of our auxiliary problem

$$
-\ln \Psi-1+\lambda(\mathrm{r})+\frac{\mu \mathrm{v}^{2}}{2}=0
$$

or

$$
\Psi=(2 \pi D)^{-\frac{3}{2}} v e^{-\frac{v^{2}}{2 D}}
$$

where

$$
D=\frac{1}{\mu}, \quad v(r)=(2 \pi D)^{\frac{3}{2}} e^{\lambda-1} \text {, }
$$

It is easy to verify, that $\nu$ is indeed the stellar number density. D is the dispersion of velocities and is independent of the coordinates. 
In agreement with the above, it is sufficient to verify the inequality (4) for functions of type (6), i.e. a Maxwellian distribution. Among such functions is also $\Psi_{0}$.

Substitute (6) into (1)

$$
L=\int \nu\left(\frac{3}{2} \ln 2 \pi \mathrm{D}+\frac{3}{2}-\ln \nu\right) \mathrm{dr} .
$$

Write down also total mass and energy

$$
\begin{gathered}
M=m \int v d r, \\
H=m \int\left(\frac{3 D}{2}+\frac{\Phi}{2}\right) v d r .
\end{gathered}
$$

In (7) the terms containing $\frac{3}{2}$ and $\frac{3}{2} \ln 2 \pi$ play no role, as they vanish in variation.

Suppose that $\nu$ is the density of an inhomogeneous but incompressible fluid. Generally speaking $\nu$ does not have to have exact spherical symmetry. In such case we can displace the elements of the fluid, without changing their density, in such a way that the fluid will assume a spherically symmetrical configuration with the density monotonically increasing towards the center. During such a displacement, L, M and $\mathrm{K}$ do not change. The functions under the integral signs in (7) and (8) do not contain coordinates explicitly and only the function $\nu$ varies.

On the other hand, $W$ decreases since the fluid mass in a spherically symmetric configuration has minimal potential energy (or, what is the same, is in the state of stable equilibrium). If we compensate for the decrease in $\mathrm{H}$ by increasing $\mathrm{D}$, $\mathrm{L}$ increases. In this fashion, it is possible to replace an arbitrary stellar number density by a spherically symmetrical one, while $\mathrm{L}$ increases. From this it can be seen that the maximum of L existing for symmetrical configurations, has to remain maximal also with respect to more general, non-symmetrical configurations. In the following, we can limit ourselves to $v$ depending on $r=\sqrt{\left(x^{2}+y^{2}+z^{2}\right)}$ on $1 y$.

We shall find now the variation of entropy with an accuracy up to terms of second order

$$
\begin{aligned}
& \delta \mathrm{L}=\iiint\left\{\nu\left[\frac{3}{2} \frac{\delta \mathrm{D}}{\mathrm{D}}-\frac{3}{4}\left(\frac{\delta \mathrm{D}}{\mathrm{D}}\right)^{2}\right\}+\frac{3}{2} \delta \nu \frac{\delta \mathrm{D}}{\mathrm{D}}+\frac{3}{2} \delta \nu \ln \mathrm{D}\right. \\
& \left.-(1 \mathrm{n} \nu+1) \delta \nu-\frac{(\delta \nu)^{2}}{2 \nu}\right\} \mathrm{dx} d \mathrm{~d} \mathrm{~d} .
\end{aligned}
$$

Also, the variations of $M$ and $H$ must be equated to zero: 


$$
\begin{aligned}
& \delta M=m \iiint \delta \nu \mathrm{dxdydz}=0 \\
& \delta \mathrm{H}=\frac{3 \mathrm{~m}}{2} \iiint(\nu \delta \mathrm{D}+\mathrm{D} \delta \nu+\delta \mathrm{D} \delta \nu) \mathrm{dx} \mathrm{dy} \mathrm{d} z \\
& +\frac{\mathrm{m}}{2} \iiint(2 \Phi \delta \nu+\delta \Phi \delta \nu) \mathrm{dx} d y \mathrm{dz}=0
\end{aligned}
$$

In deriving formula (12) we have used the reciprocity relation known from potential theory

$$
\iiint \Phi \delta \nu \mathrm{dx} d y \mathrm{~d} z=\iiint \nu \delta \Phi \mathrm{dx} \mathrm{dy} \mathrm{d} z
$$

For a stationary state $\nu$ has to satisfy a relationship which can be obtained by substitution of expression (6) - in the form

$$
\Psi_{0}=(2 \pi \mathrm{D})^{-\frac{3}{2}} v \mathrm{e}^{\frac{\Phi}{\mathrm{D}}} \mathrm{e}^{-\frac{1}{\mathrm{D}}\left(\frac{\mathrm{v}^{2}}{2}+\Phi\right)}
$$

-into the Boltzmann equation.

Here, the last coefficient is a function of the energy integrals of each star separately; consequently, it cancels out after substitution into Boltzmann's equation. The remaining function of coordinates satisfies Boltzmann's equation and reduces to a constant. As a result, for stationary systems

$$
\nu=c e^{-\frac{\Phi}{D}}\left(D=\sigma^{2}\right) \text {. }
$$

We multiply the variations $\delta M$ and $\delta H$ by Lagrange multipliers and combine with $\delta \mathrm{L}$ :

$$
\begin{aligned}
& \delta \mathrm{L}-\frac{\delta \mathrm{H}}{\mathrm{mD}}+\left(\ln \mathrm{c}+\frac{5}{2}-\frac{3}{2} \ln \mathrm{D}\right) \frac{\delta \mathrm{M}}{\mathrm{m}}= \\
& =\iiint\left[\frac{3 \ln \mathrm{D}}{2}-(\ln v+1)-\left(\frac{3}{2}+\frac{\Phi}{\mathrm{D}}\right)\right. \\
& \left.+\left(\operatorname{lnc}+\frac{5}{2}-\frac{3}{2} \ln \mathrm{D}\right)\right] \delta v \mathrm{dx} \mathrm{dy} \mathrm{d} z+ \\
& + \text { Second order terms }
\end{aligned}
$$

Here the values of the Lagrange multipliers were already substituted, making the first order terms in $\delta \nu$ and $\delta \mathrm{D}$ vanish. This may be easily verified, having in mind (13). The remaining terms of second order give

$$
\delta \mathrm{L}=-\iiint\left[\frac{(\delta \nu)^{2}}{2 \nu}+\frac{3}{4 \mathrm{D}^{2}}(\delta \mathrm{D})^{2}+\frac{\delta \nu \delta \Phi}{2 \mathrm{D}}\right] \mathrm{dx} \mathrm{dy} \mathrm{d} z \text {. }
$$


We shall solve (12) for $\delta D$ to be substituted into (14), remembering that $\mathrm{D}$ and $\delta \mathrm{D}$ are independent of the coordinates.

$$
\delta D=-\frac{2}{3} \frac{\iiint \Phi \delta \nu d x d y d z}{\iiint \nu d x d y d z}+\ldots
$$

The remaining terms are not needed, since they yield terms of higher order, for the substitution into (14).

$$
\delta L=-2 \pi \int_{0}^{R}\left[\frac{(\delta \nu)^{2}}{2 \nu}+\frac{\delta \nu \delta \Phi}{D}\right] r^{2} d r-\frac{4 \pi\left(\int_{0}^{R} r^{2} \Phi \delta \nu d r\right.}{3 D^{2} \int_{0}^{R} r^{2} v d r} .
$$

where $\delta v$ must satisfy the additional condition (11), which is equivalent to

$$
\int_{0}^{\mathrm{R}} \mathrm{r}^{2} \delta v d r=0 .
$$

Let us now introduce a variable

$$
\mathrm{T}=\frac{\mathrm{r}^{2}}{4 \pi \mathrm{mG}} \cdot \frac{\mathrm{d} \delta \Phi}{\mathrm{dr}}
$$

and notice that in agreement with Poisson's equation

$$
4 \pi \mathrm{mG} \delta \nu=\frac{1}{\mathrm{r}^{2}} \cdot \frac{\mathrm{d}}{\mathrm{dr}}\left(\mathrm{r}^{2} \frac{\mathrm{d} \delta \Phi}{\mathrm{dr}}\right) .
$$

It follows that

$$
\delta v=\frac{1}{\mathrm{r}^{2}} \cdot \frac{\mathrm{dT}}{\mathrm{dr}}
$$

which, considering (17), gives $T(R)=T(0)=0$, where $T(r=0)=0$ is of third order in $r$.

In (16) integrate by parts: 


$$
\begin{aligned}
& \int_{0}^{\mathrm{R}} \mathrm{r}^{2} \delta \nu \delta \Phi \mathrm{dr}=\int_{0}^{\mathrm{R}} \delta \Phi \frac{\mathrm{dT}}{\mathrm{dr}} \mathrm{dr}=-4 \pi \mathrm{mG} \int_{0}^{\mathrm{R}} \frac{\mathrm{T}^{2}}{\mathrm{r}^{2}} \mathrm{dr}, \\
& \int_{0}^{\mathrm{R}} \mathrm{r}^{2} \Phi \delta \nu \mathrm{dr}=\int_{0}^{\mathrm{R}} \Phi \frac{\mathrm{dT}}{\mathrm{dr}} \mathrm{dr}=-\int_{0}^{\mathrm{R}} \mathrm{T} \frac{\mathrm{d} \Phi}{\mathrm{dr}} \mathrm{dr},
\end{aligned}
$$

and (16) becomes

$$
\begin{aligned}
& \delta L=-2 \pi \int_{0}^{R}\left[\frac{1}{r^{2} \nu}\left(\frac{d T}{d r}\right)^{2}-\frac{4 \pi m G}{D} \cdot \frac{T^{2}}{r^{2}}\right] d r \\
& -\frac{4 \pi\left(\int_{0}^{R} T \frac{d \Phi}{d r} d r\right)^{2}}{3 D^{2} \int_{0}^{R} r^{2} \nu d r}
\end{aligned}
$$

Consider the function

$$
\phi(r)=r^{2} \frac{d}{d r}\left(r \frac{d \Phi}{d r}\right)=r^{2} \frac{d}{d r}(r F)=r^{3} 4 \pi m G \nu-r^{2} F,
$$

where $F(r)$ is the strength of the gravitational field. The asymptotic behavior of $\mathrm{F}$ is well known ${ }^{6}$

$$
\lim r F=2 D,
$$

where the approach to the limit is oscillatory. Thus, $\phi(r)$ has an infinite number of zeros, which we arrange in ascending order: $r_{0}=0, r_{1}, r_{2}, \ldots$

We will show that for $R<r_{1}$ the first integral in (19) and consequently the whole expression is always negative. For this purpose we first establish that $\phi(r)$ satisfies the differential equation

$$
\frac{d}{d r}\left(\frac{1}{r^{2} v} \cdot \frac{d \phi}{d r}\right)+\frac{4 \pi m G}{D} \cdot \frac{\phi}{r^{2}}=0
$$


We verify (21)

$$
\frac{d \phi}{d r}=4 \pi m G \frac{d}{d r} \quad\left(r^{3} \cdot v\right)-4 \pi m G r^{2} v=4 \pi m G \quad\left(r^{3} \frac{d v}{d r}+2 r^{2} v\right) .
$$

But from (13) easily follows

$$
\frac{1}{v} \cdot \frac{\mathrm{d} v}{\mathrm{dr}}=-\frac{\mathrm{F}}{\mathrm{D}}
$$

Consequently,

$$
\frac{1}{\mathrm{r}^{2} \nu} \cdot \frac{\mathrm{d} \phi}{\mathrm{dr}}=-\frac{4 \pi m G}{\mathrm{D}} \mathrm{rF}+8 \pi m G \text {, }
$$

from which we deduce the validity of (21). equation

Take $R=r_{1}$. Then $\phi$ may be considered as an eigenfunction of the

$$
\frac{d}{d r}\left(\frac{1}{r^{2} v} \cdot \frac{d \phi}{d r}\right)+\frac{4 \pi m G}{D} \lambda \frac{\phi}{r^{2}}=0,
$$

It is the first eigenfunction in view of its constant sign, and the first eigenvalue $\lambda=1$. This corresponds to the value of the minimum

$$
\min \frac{\int_{0}^{R} \frac{1}{r^{2} v}\left(\frac{d T}{d r}\right)^{2} d r}{\int_{0}^{R} \frac{4 \pi m G}{D} \cdot \frac{T^{2}}{r^{2}} d r},
$$

which is equal to unity, Q.E.D.

Take further $R=r_{2}$. Then $\lambda=1$ is the second eigenvalue of (23) with the same $\phi$. We shall prove that (19) can assume positive values. Let $\mathrm{T}$ be subject to the additional constraint

$$
\int_{0}^{R} T \frac{d \Phi}{d r} d r=0
$$

With this supplementary condition, the minimum (24) - in agreement with Courant's theorem ${ }^{7}$ - is between the first and the second eigenvalue, and therefore is smaller than 1 . The function $T$ for which this minimum is attained makes $\delta$ L positive, Q.E.D. function

For a more exact investigation of expression (19) replace $\mathrm{T}$ by the 


$$
\mathrm{T}_{0}=\mathrm{r}^{2} \frac{\mathrm{d}}{\mathrm{dr}}(\mathrm{rF})+\mu \mathrm{r}^{2} \mathrm{~F},
$$

where the parameter $\mu$ is selected so that the boundary condition $\mathrm{T}_{0}(\mathrm{R})=0$ is satisfied,

$$
\mu=-\frac{\left(\frac{d(r F)}{d r}\right)_{r=R}}{F(R)} .
$$

Integrating per parts and taking into consideration that by (25), $\mathrm{T}$ has a zero of third order at $r=0$, we obtain

$$
\begin{aligned}
& \int_{0}^{\mathrm{R}} \frac{1}{\mathrm{r}^{2} v}\left(\frac{\mathrm{dT}_{0}}{\mathrm{dr}}\right)^{2} \mathrm{dr}=-\int_{0}^{\mathrm{R}} \mathrm{T}_{0} \frac{\mathrm{d}}{\mathrm{dr}}\left(\frac{1}{\mathrm{r}^{2} v} \cdot \frac{\mathrm{dT}_{0}}{\mathrm{dr}}\right) \mathrm{dr}, \\
& \frac{d}{d r}\left(\frac{1}{r^{2} v} \cdot \frac{d_{0}}{d r}\right)+\frac{4 \pi m G}{D} \cdot \frac{T_{0}}{r}=\mu\left\{\frac{d}{d r}\left[\frac{1}{r^{2} v} \cdot \frac{d\left(r^{2} F\right)}{d r}\right]\right. \\
& \left.+\frac{4 \pi m G}{D} r F\right\}=\frac{4 \pi m G}{D} \mu F \text {, } \\
& \delta \mathrm{L}=2 \pi \int_{0}^{\mathrm{R}} \mathrm{T}_{0} \frac{4 \pi m G}{\mathrm{D}} \mu \mathrm{Fdr}-\frac{4 \pi\left(\int_{0}^{\mathrm{R}} \mathrm{T}_{0} \mathrm{~F} \mathrm{dr}\right)^{2}}{3 \mathrm{D}^{2} \int_{0}^{\mathrm{R}} \mathrm{r}^{2} \nu \mathrm{dr}}= \\
& =\left(\frac{4 \pi m G}{D} \mu-\frac{20^{\int^{R}} \mathrm{~T}_{0} \mathrm{Fdr}}{3 \mathrm{D}^{2} \int_{0}^{\mathrm{R}} \mathrm{r}^{2} v \mathrm{dr}}\right) 2 \pi \int_{0}^{\mathrm{R}} \mathrm{T}_{0} \mathrm{~F} \mathrm{dr} .
\end{aligned}
$$

Notice that for $R \rightarrow 0 \quad \mu \rightarrow-2$, since near the center, the gravitational force may beconsidered proportional to the distance from the center. Consequently, for $0<\mathrm{R}<\mathrm{r}_{1}$ we have $\mu<0$. If we left out the last integral in (19) then in place of (26) we would get

$$
\frac{4 \pi m G}{D} \mu 2 \pi \int_{0}^{R} T_{0} F d r \text {, }
$$

but this expression - as was already shown - is negative for $0<\mathrm{R}<\mathrm{r}_{1}$ and consequently the integral itself 


$$
\int_{0}^{\mathrm{R}} \mathrm{T}_{0} \mathrm{~F} \mathrm{dr}>0
$$

Let us gradually increase $R$, beginning with value $r_{1}$. Then for some $R_{k}$ lying between $r_{1}$ and $r_{2}$, the expression in parentheses in (26) changes its sign from $(-)$ to $(+)$ (detailed calculation shows this). Since in the interval $\left(r_{1}, R_{k}\right)$ we have $\mu>0$, the inequality (27) is still valid in this interval.

From (26) it follows that for $R>R_{k} \quad \delta L$ can assume positive values. It remains to prove that when $R=R_{k}$ (and hence also when $R<R_{k}$ ) $\delta L$ cannot assume positive values. To show this represent an arbitrary $\mathrm{T}$ by

$$
\mathrm{T}=\tau \mathrm{T}_{0}+\mathrm{T}_{1}
$$

for $R=R_{k}$, where $T_{1}$ satisfies the constraint

$$
\int_{0}^{\mathrm{R}_{\mathrm{k}}} \mathrm{T}_{1} \psi \mathrm{dr}=0 \text {, }
$$

and $\mathrm{T}_{0}$ is determined by (25). Obvious $1 \mathrm{y}, \mathrm{T}_{1}(0)=\mathrm{T}_{1}(\mathrm{R})=0$

Here, $\psi$ is the first eigenfunction of equation (23) with boundary conditions $\psi(0)=\psi\left(R_{k}\right)=0$. From (28) and (29) the value of $\tau$ is easily found

$$
\tau=\frac{\int_{0}^{R_{k}} \mathrm{~T} \psi d r}{\int_{0}^{R_{k}} T_{0} \psi d r} .
$$

The substitution $\mathrm{T}_{1}=\mathrm{T}-\tau \mathrm{T}_{0}$ in (29) shows that (29) is satisfied. The consistency of ${ }^{1}(28)$ will be proven if we can establish that the denominator in (30) does not vanish.

The minimum of (24) with the constraint

$$
\int_{0}^{\mathrm{R}} \mathrm{T} \psi \mathrm{dr}=0
$$

is indeed equal to the second eigenvalue of the equation (23); this value is equal to unity when $R=r_{2}$ and consequently is larger than 1 when $R=R_{k}<r_{2}$. It follows that if $T_{0}$ were orthogonal to $\psi$, then (19) with $\mathrm{T}=\mathrm{T}_{0}$ would be negative, but this contradicts the choice of $\mathrm{R}_{\mathrm{k}}$. We substitute (28) into (19):

1) The terms of the first and the second order in $\tau$ are 


$$
\begin{gathered}
-2 \pi \tau \int_{0}^{\mathrm{R}} \frac{1}{\mathrm{r}^{2} v} \cdot \frac{\mathrm{dT}_{0}}{\mathrm{dr}}\left(\tau \frac{\mathrm{dT}_{0}}{\mathrm{dr}}+2 \frac{\mathrm{dT}_{1}}{\mathrm{dr}}\right) \mathrm{dr} \\
+2 \pi \tau \frac{4 \pi \mathrm{mG}}{\mathrm{D}} \int_{0}^{\mathrm{R}} \frac{\mathrm{T}_{0}\left(\tau \mathrm{T}_{0}+2 \mathrm{~T}_{1}\right)}{\mathrm{r}^{2}} \mathrm{dr} \\
-\frac{4 \pi \tau}{3 \mathrm{D}^{2}} \cdot \frac{0^{\delta^{\mathrm{R}}\left(2 \mathrm{~T}_{1}+\tau \mathrm{T}_{0}\right) \mathrm{F} \mathrm{dr}{ }_{0}{ }^{\mathrm{R}} \mathrm{T}_{0} \mathrm{Fdr}}}{0^{\mathrm{R}} \mathrm{r}^{2} \nu \mathrm{dr}}
\end{gathered}
$$

Integration by parts as in the derivation of (26) gives

$$
\begin{aligned}
& -\int_{0}^{\mathrm{R}} \frac{1}{\mathrm{r}^{2} \nu} \cdot \frac{\mathrm{dT}_{0}}{\mathrm{dr}}\left(\tau \frac{\mathrm{dT}_{0}}{\mathrm{dr}}+2 \frac{\mathrm{dT}_{1}}{\mathrm{dr}}\right) \mathrm{dr}= \\
& =\int_{0}^{\mathrm{R}}\left(\tau \mathrm{T}_{0}+2 \mathrm{~T}_{1}\right) \frac{\mathrm{d}}{\mathrm{dr}}\left(\frac{1}{\mathrm{r}^{2} v} \cdot \frac{\mathrm{dT}_{0}}{\mathrm{dr}}\right) \mathrm{dr} .
\end{aligned}
$$

Consequent $1 \mathrm{y}$

$$
\begin{aligned}
& -\int_{0}^{\mathrm{R}} \frac{1}{\mathrm{r}^{2}} \cdot \frac{\mathrm{dT}_{0}}{\mathrm{dr}}\left(\tau \frac{\mathrm{dT}_{0}}{\mathrm{dr}}+2 \frac{\mathrm{dT}_{1}}{\mathrm{dr}}\right) \mathrm{dr} \\
& +\frac{4 \pi \mathrm{mG}}{\mathrm{D}} \int_{0}^{\mathrm{R}} \frac{\mathrm{T}_{0}\left(\tau \mathrm{T}_{0}+2 \mathrm{~T}_{1}\right)}{\mathrm{r}^{2}} \mathrm{dr}= \\
& =\int_{0}^{\mathrm{R}}\left(\tau \mathrm{T}_{0}+2 \mathrm{~T}_{1}\right) \frac{4 \pi \mathrm{mG}}{\mathrm{D}} \mu \mathrm{F} \mathrm{dr} .
\end{aligned}
$$

Taking into account the definition of $R_{k}$, we can see that the sum of the terms of the first and second order in $\tau$ vanishes.

2) The remaining terms do not contain $\tau$; these are obtained from (19) by substituting $\mathrm{T}_{1}$ for $\mathrm{T}$. The resulting expression is negative, which follows by inspection of the minimum (24) in view of (29).

Proving in this manner the critical character of the value $R=R_{k}$, we shall now write the equation for $\mathrm{R}_{\mathrm{k}}$ explicitly. In particular, 
replacing $\mu$ by its definition and replacing $\mathrm{T}_{0}$ by the definition (25), we deduce from the vanishing of the parenthesized term in (26) for $R=R_{k}$ that

$$
\begin{aligned}
& 6 \pi m G D(4 \pi m G R v-F) \int_{0}^{R} r^{2} \nu d r-4 \pi m G R \nu \int_{0}^{R} r^{2} F^{2} d r+ \\
& +4 \pi m G F \int_{0}^{R} r^{3} \nu F d r=0
\end{aligned}
$$

The integrals in equation (31) may be eliminated using

$$
\int_{0}^{R} r^{2} v d r=\frac{R^{2} F(R)}{4 \pi m G}
$$

and the remaining two integrals we integrate by parts:

$$
\begin{aligned}
& \int_{0}^{R} r^{2} F^{2} d r=\frac{R^{3}}{3}(F(R))^{2}-\frac{2}{3} \int_{0}^{R} r^{3} F F^{\prime} d r= \\
& =\frac{R^{3}}{3}(F(R))^{2}-\frac{2}{3} \int_{0}^{R} r^{3} F\left(4 \pi m G-\frac{2 F}{r}\right) d r=\frac{R^{3}(F(R))^{2}}{3} \\
& -\frac{2}{3} \int_{0}^{R} 4 \pi m G r^{3} v F d r+\frac{4}{3} \int_{0}^{R} r^{2} F^{2} d r,
\end{aligned}
$$

whence

$$
\int_{0}^{R} r^{2} F^{2} d r=-R^{3}(F(R))^{2}+8 \pi m G \int_{0}^{R} r^{3} v F d r
$$

From (22) follows the relation

Therefore

$$
\begin{aligned}
& \int_{0}^{R} r^{3} v F d r=-D \int_{0}^{R} r^{3} v^{\prime} d r=-D\left(R^{3} v(R)-3 \int_{0}^{R} r^{2} v d r\right)= \\
& =-D\left(R^{3} v(R)-\frac{3}{4 \pi m G} R^{2} F(R)\right) .
\end{aligned}
$$

$$
\int_{0}^{R} r^{2} \mathbf{F}^{2} d x=-\mathbf{R}^{3}(\boldsymbol{F}(\mathrm{R}))^{2}-8 \pi m G \mathrm{DR}^{3} \nu(\mathrm{R})+6 \mathrm{DR}^{2} F(\mathrm{R}) .
$$


Substituting the derived expressions for the integrals in (31), we have

$$
\begin{aligned}
& \frac{3}{2}(4 \pi m G R \nu-F) D R^{2} F-4 \pi m G R \nu\left(-R^{3} F^{2}-8 \pi m G D R^{3} \nu+6 D R^{2} F\right) \\
& -4 \pi m G D R^{3} \nu F+3 D R^{2} F^{2}=0 .
\end{aligned}
$$

On the other hand, combining (13) with Poisson's equation, we obtain

$$
\frac{1}{\mathrm{r}^{2}} \cdot \frac{\mathrm{d}}{\mathrm{dr}} \cdot\left(\mathrm{r}^{2} \frac{\mathrm{d} \ln \nu}{\mathrm{dr}}\right)=-\frac{4 \pi m G}{\mathrm{D}} \nu \text {. }
$$

The order of equation (36) can be reduced in several different ways ${ }^{6}$. Thus, e.g., we introduce new variables

$$
\mathrm{x}=-\mathrm{r} \frac{\mathrm{d} \ln v}{\mathrm{dr}} \text { and } \mathrm{y}=\mathrm{r} \frac{\mathrm{dx}}{\mathrm{dr}} \text {. }
$$

Clearly, by its definition $x>0$. We substitute $x$ into (22) and get

$$
\mathrm{F}=\frac{\mathrm{Dx}}{\mathrm{r}} \text {. }
$$

Substitution of $\mathrm{x}$ in the left-hand side of (36) yields

$$
\frac{1}{r^{2}} \frac{d}{d r}(r x)=\frac{4 \pi m G}{D} v
$$

or

$$
\frac{4 \pi m G}{D} v=\frac{x+y}{r^{2}}
$$

Next, we take the logarithm of (39) and differentiate with respect to $r$, keeping in mind the transformation (37):

$$
\frac{\frac{d x}{d r}+\frac{d y}{d r}}{x+y}-\frac{2}{r}=\frac{d \ln v}{d r}=-\frac{x}{r} \text {, }
$$

Substituting $y$ as given by (37) and solving for $\frac{d y}{d x}$, we have

$$
\frac{d y}{d x}=\frac{2 x+y+x-x y}{y}
$$

The initial conditions are obtained by taking $\mathrm{r}=0$; then $\mathrm{x}=0, \mathrm{y}=0$ and $\mathrm{dy} / \mathrm{d} \mathrm{x}=2$. The previously introduced variable $\phi$ differs from $y$ only by the coefficient $r$. Therefore, the values of radius $0, r_{1}, r_{2} \ldots$ correspond to the points of intersection of integral curve (40) with the axis of abscisses $0(0) 0,, A_{1}\left(x_{1}, 0\right), A_{2}\left(x_{2}, 0\right) \ldots$ The integral curve passes from the point 0 from left to the right in the upper half-plane 
while in the lower half-plan, from $A_{1}$ to $A_{2}$, from right to the left (since for $y=0$ dy changes sign, passing through infinity) and further continues to turn around the point $\mathrm{C}(2,0)$ as around a focus.

We shall utilize now (38) and (39) in the transformation (35):

$$
\begin{aligned}
& \frac{3}{2} D^{2} R x \frac{D y}{R}-\frac{D(x+y)}{R}\left[-D^{2} R x^{2}-2 D^{2} R(x+y)+7 D^{2} R x\right]+ \\
& +3 D^{3} x^{2}=0,
\end{aligned}
$$

from which after obvious algebra we get

$$
y=\frac{3 x-2 x^{2} \pm x \sqrt{4 x^{2}-44 x+73}}{8}
$$

In the region of interest $0<\mathrm{x}<\mathrm{x}_{1}$ the curve represented by (41) has the shape of a closed loop, passing, in particular, through the points 0 and $C$. Therefore, the point corresponding to $R_{k}$, i.e. the point of intersection of curves (40) and (41) necessarily exists. It must be located on the section of the integral curve between $A_{1}$ and $A_{2}$. Numerical computation gives the coordinates of the point of intersection: $x=+2.031 ; y=-0.364$. From the values of $x$ and $y$ we go back to $v$ :

$$
\frac{\mathrm{d} \ln \nu}{\mathrm{dr}}=-\frac{\mathrm{x}}{\mathrm{r}}, \quad \ln \frac{\nu(\mathrm{r})}{\nu(0)}=-\int_{0}^{\mathrm{r}} \frac{\mathrm{x}}{\mathrm{r}} \mathrm{dr}=-\int_{0}^{\mathrm{x}} \frac{\mathrm{x} \mathrm{dx}}{\mathrm{y}} .
$$

Integration with $r_{=} R_{k}$ yields

$$
\frac{v\left(R_{k}\right)}{\nu(0)}=\frac{1}{709}
$$

Thus, we have proven that the density in the center of the main body of a star system with Maxwellian distribution of velocities, cannot exceed the density on the boundary of the main body more than 709 times.

On the other hand, by equation (39) we have

$$
\begin{aligned}
& \mathrm{R}_{\mathrm{k}}^{2}=\frac{(\mathrm{x}+\mathrm{y}) \mathrm{D}}{4 \pi \mathrm{mG} v}=\frac{1,667 \cdot 2^{2} \cdot 709}{4 \pi \mathrm{mG} v(0)}, \\
& \mathrm{R}_{\mathrm{k}}=\frac{9}{\sqrt{\mathrm{d}^{\circ} \mathrm{G}}},
\end{aligned}
$$

where $d$ is mean density of the substance in the center and $R_{k}$ defines the region which tends to establish within itself a Maxwellian distribution.

The fact that $\delta \mathrm{L}$ may assume positive values for sufficiently large $R\left(R>R_{k}\right)$ means that Maxwellian distribution does not give a maximum of the entropy and that a phase distribution more probable (in the sense of Boltzmann's formula) than a stationary Maxwellian distribution is 
possible. What has to be the nature of the evolution of a system if at the initial moment of time it finds itself in such an "excessively probable" state? In the first place, the system will not be approaching the Maxwell's distribution but going further away from it, on account of Boltzmann's theorem. Secondly, when the entropy reaches a certain limit the system will become non-stationary. We shall prove this last assertion.

Let us divide the phase volume bounded in physical space by sphere $\mathrm{x}^{2}+\mathrm{y}^{2}+\mathrm{z}^{2} \leq \mathrm{R}$ into two regions such that in the first region

$$
\Psi \geq \mathrm{e}^{-\frac{v^{2}}{2 h}-1},
$$

and in the second region

$$
\Psi<e^{-\frac{v^{2}}{2 h}-1} \leq \frac{1}{e}
$$

where $h$ is an arbitrary positive constant.

Now, estimate the entropy

$$
\begin{aligned}
& \mathrm{L}=-\iint \Psi \ln \Psi \mathrm{dr} \mathrm{dv}-\iint \Psi \ln \Psi \mathrm{dr} \mathrm{dv} \leq \\
& \text { region I region II } \\
& \leq \iint_{\text {region } I} \Psi\left(1+\frac{v^{2}}{2 h}\right) d r d v+\iint\left(1+\frac{v^{2}}{2 h}\right) e^{-\frac{v^{2}}{2 h}-1} d r d v .
\end{aligned}
$$

The substitution in the second integral was made on the basis of the fact that $x \ln x$ is a decreasing function for $x<\frac{1}{e}$.

We may strengthen the inequalities by integrating both integrals throughout the whole phase volume.

or

$$
\mathrm{L} \leq \frac{\mathrm{M}}{\mathrm{m}}+\frac{\mathrm{K}}{\mathrm{hm}}+\frac{5}{2 \mathrm{e}}(2 \pi \mathrm{h})^{3 / 2} \frac{4}{3} \pi \mathrm{R}^{3}
$$

$$
\mathrm{K} \geq \mathrm{hmL}-\mathrm{hM}-\frac{5 \mathrm{hm}}{2 \mathrm{e}}(2 \pi \mathrm{h})^{3 / 2} \frac{4}{3} \pi \mathrm{R}^{3} \text {. }
$$

It is clear from these relations that when the entropy increases, beginning at some moment, the inequality $\mathrm{K}>-\mathrm{H}$, i.e. $2 \mathrm{~K}+\mathrm{W}>0$ will hold. In that case virial theorem shows that the system must disintegrate or else expand beyond the initial boundaries, which is what we wanted to prove.

Finally, we will show that in our problem the entropy maximum can only be relative and not absolute. For a special phase distribution we take the following. Let mass $\alpha \mathrm{M}$ be uniformly distributed inside the sphere $N_{1}$ with radius $\rho_{1}$, with a uniform distribution of velocities. Let the velocities have upper limit $C_{1}$. Let the second part of the total star mass $B M$ be distributed throughout sphere $\mathrm{N}_{2}$ of radius $\rho_{2}$ in 
analogous way, with maximum velocity $c_{2}$. Let the distance between the centers of $\mathrm{N}_{1}$ and $\mathrm{N}_{2}$ be $\rho_{12}$, the spheres being non-intersecting. Outside $N_{1}, N_{2}$ the phase density is equal to zero. By the law of conservation of mass $\alpha+\beta=1$.

The corresponding phase densities are equal to

$$
\Psi_{1}=\frac{\alpha M}{m \frac{4}{3} \pi \rho_{1}^{3} \frac{4}{3} \pi c_{1}^{3}} \quad, \quad \Psi_{2}=\frac{\beta M}{m \frac{4}{3} \pi \rho \frac{4}{2} \pi c_{2}^{3}}
$$

The entropy is

$$
\begin{aligned}
& L=-\frac{\alpha M}{m} \ln \Psi_{1}-\frac{\beta M}{m} \ln \Psi_{2}=-\frac{M}{m}(\alpha \ln \alpha+\beta \ln \beta)+ \\
& +\frac{3 M}{m}\left(\beta \ln c_{2} \rho_{2}+\alpha \ln c_{1} \rho_{1}\right) .
\end{aligned}
$$

The total energy, after some algebra, is seen to be

$$
\mathrm{H}=-\frac{3 G \alpha^{2} M^{2}}{5 \rho_{1}}-\frac{3 G \beta^{2} M^{2}}{5 \rho_{2}}-\frac{\alpha \beta M^{2}}{\rho_{12}}+\frac{3}{10}\left(\alpha M c_{1}^{2}+\beta M c_{2}^{2}\right) .
$$

$c_{2}, \rho_{1}$ and also the quantity $f=-\beta$ ln $\left(c_{2} \rho_{2}\right)$ will be considered constant. On the other hand, let $\rho_{2}$ be infinitesimally small. The remaining parameter $c_{1}$ will be chosen such that (43) is always satisfied, i.e.

$$
c_{1}=\sqrt{\frac{1}{\alpha}\left[\frac{10}{3 M}\left(H+\frac{\alpha \beta M^{2}}{\rho_{12}}\right)+\frac{2 G \alpha^{2} M}{\rho_{1}}-\beta c_{2}^{2}+\frac{2 G f^{2} M}{\rho_{2}\left(\ln c_{2} \rho_{2}\right)^{2}}\right]} \text {. }
$$

In the limit that $\rho_{2} \rightarrow 0, \beta \rightarrow 0, \alpha \rightarrow 1$, we have $c_{1} \rightarrow+\infty$.

In (42), all terms with the exception of the last one tend to finite limits :

$$
\alpha \ln \alpha \rightarrow 0, \beta \text { In } \beta \rightarrow 0, \beta \text { ln } c_{2} \rho_{2}=-\mathrm{f},
$$

but the increase of $c_{1}$ has as a consequence the increase of $L$ beyond any limit, i.e. there is no absolute maximum.

In conclusion the author expresses his gratitude to Professor

K. F. Ogorodnikov for his great help in the formulation of this article.

\section{References}

1. Agekyan, T.A. Vestnik L.G.U. No. 1 (1962)

2. Carleman, T. Problemes Mathematiques dans la theorie cinetique des gaz (Uppsala: Almqvist \& Wiksells, 1957)

3. Ogorodnikov, K.F. Dynamics of Stellar Systems (New York: Pergamon, 1965)

4. Ambartsumian, V.A. transl. in Appendix I in these proceedings. (1938)

5. Lavrent'ev, M.A. \& Lyusternik, L.A. Kurs variatsionnovo ischisteniya

(Course on variational calculus; Gostekhizdat, 1950)

6. Chandrasekhar, S. An Introduction to the Study of Stellar Structure (Dover, 1967)

7. Courant, R. \& Hilbert, D. Methods of Mathematical Physics (Wiley, 1962) 